\title{
A Nonlinear $r(x)$-Kirchhoff Type Hyperbolic Equation: Stability Result and Blow up of Solutions with Positive Initial Energy
}

\author{
Mohammad Shahrouzi $^{1 *}$, Jorge Ferreira ${ }^{2}$
}

\begin{abstract}
In this paper we consider $r(x)$-Kirchhoff type equation with variable-exponent nonlinearity of the form

$$
u_{t t}-\Delta u-\left(a+b \int_{\Omega} \frac{1}{r(x)}|\nabla u|^{r(x)} d x\right) \Delta_{r(x)} u+\beta u_{t}=|u|^{p(x)-2} u,
$$

associated with initial and Dirichlet boundary conditions. Under appropriate conditions on $r($.$) and p($.$) , stability$ result along the solution energy is proved. It is also shown that regarding arbitrary positive initial energy and suitable range of variable exponents, solutions blow-up in a finite time.
\end{abstract}

Keywords: blow-up, Kirchhoff equation, stability result, variable exponents 2010 AMS: 35B35, 35B44, 35L72, 35N10

\section{${ }^{1}$ Department of Mathematics, Jahrom University, Jahrom Iran, P.O.Box: 74137-66171}

${ }^{2}$ Department of Mathematics, Federal University Fluminense, Volta Redonda, Brazil

*Corresponding author: mshahrouzi@jahromu.ac.ir

Received: 25 May 2021, Accepted: 13 December 2021, Available online: 27 December 2021

\section{Introduction}

Let $\Omega$ be a bounded domain of $R^{n}(n \geq 1)$ with a smooth boundary $\partial \Omega$. Consider the following $r(x)-$ Kirchhoff type hyperbolic boundary value problem

$$
\begin{aligned}
& u_{t t}-\Delta u-\left(a+b \int_{\Omega} \frac{1}{r(x)}|\nabla u|^{r(x)} d x\right) \Delta_{r(x)} u+\beta u_{t}=|u|^{p(x)-2} u, \quad(x, t) \in \Omega \times(0, \infty), \\
& u(x, t)=0 \quad(x, t) \in \partial \Omega \times(0, \infty), \\
& u(x, 0)=u_{0}(x), u_{t}(x, 0)=u_{1}(x), x \in \Omega,
\end{aligned}
$$

where $a, b, \beta$ are positive constants and $\Delta_{r(x)}$ is called $r(x)$-Laplace operator defined as

$$
\Delta_{r(x)} u=\operatorname{div}\left(|\nabla u|^{r(x)-2} \nabla u\right) .
$$


Here, we have the following condition on the variable exponents:

(A1) the exponents $r($.$) and p($.$) are given measurable functions on \bar{\Omega}$ such that:

$$
\begin{aligned}
& 2<r_{1} \leq r(x) \leq r_{2}<\infty, \\
& 2<p_{2} \leq p(x) \leq p_{2}<\infty,
\end{aligned}
$$

with

$$
\begin{gathered}
r_{1}:=\operatorname{essinf}_{x \in \bar{\Omega}} r(x), r_{2}:=\operatorname{esssup}_{x \in \bar{\Omega}} r(x), \\
p_{1}:=\operatorname{essinf}_{x \in \bar{\Omega}} p(x), \quad p_{2}:=\operatorname{esssup}_{x \in \bar{\Omega}} p(x) .
\end{gathered}
$$

Before going any further, it is worth pointing out some results about the Kirchhoff-type equations. Kirchhoff equation

$$
u_{t t}-M\left(\int_{\Omega}\left|\nabla_{x} u\right|^{2} d x\right) \Delta_{x} u=f(x, t)
$$

where $M(s)=a s+b, a, b>0$, was proposed by Kirchhoff [1] as an extension of the classical D'Alembert's wave equation for free vibrations of elastic strings. In the last decade many papers in the literature have investigated the existence of solutions and blow-up results to the Kirchhoff-type problem. For example, Matsuyama and Ikehata [2] considered the following initial-boundary value problem

$$
\begin{gathered}
u_{t t}-M\left(\|\nabla u(t)\|_{2}^{2}\right) \Delta u+\delta\left|u_{t}\right|^{p-1} u_{t}=\mu|u|^{q-1} u, \quad t \geq 0, x \in \Omega, \\
u(0, x)=u_{0}(x), u_{t}(0, x)=u_{1}(x), \quad x \in \Omega, \\
\left.u(t, x)\right|_{\partial \Omega}=0, \quad t \geq 0 .
\end{gathered}
$$

They proved a global solvability in the class $H^{2} \times H_{0}^{1}$ and energy decay of the problem without the smallness of the initial data in a certain sense. Ono [3] investigated the global existence, decay properties, and blow-up of solutions to the nonlinear Kirchhoff strings with nonlinear dissipation. Pişkin [4] considered the initial-boundary value problem for the following extensible beam equation with nonlinear damping and source terms

$$
\begin{gathered}
u_{t t}+\Delta^{2} u-M\left(\|\nabla u\|^{2}\right) \Delta u+\left|u_{t}\right|^{p-1} u_{t}=|u|^{q-1} u(x, t) \in \Omega \times(0 . T), \\
u(x, 0)=u_{0}(x), u_{t}(x, 0)=u_{1}(x) \quad x \in \Omega, \\
u(x, t)=\frac{\partial}{\partial v} u(x, t)=0 \quad x \in \partial \Omega,
\end{gathered}
$$

author established the existence of the solution by Banach contraction mapping principle and the decay estimates of the solution by using Nakao's inequality. Moreover, under suitable conditions on the initial datum, the blow up of solutions in finite time has been proved.

In another study, the following initial boundary value problem for a Kirchhoff type plate equation has been considered by Zhou [5]:

$$
\begin{gathered}
u_{t t}+\alpha \Delta^{2} u-a \Delta u-b\left(\int_{\Omega}|\nabla u|^{2} d x\right)^{\gamma} \Delta u+\lambda u_{t}=\mu|u|^{p-2} u \text { in } \Omega_{T}, \\
u(x, 0)=u_{0}(x), u_{t}(x, 0)=u_{1}(x) \text { in } \Omega, \\
u(x, t)=\partial_{v} u(x, t)=0 \text { on } \Gamma .
\end{gathered}
$$

He proved the blow-up of solutions and the lifespan estimates for three different ranges of initial energy. Global existence of solutions has been proved by the potential well theory, and decay estimates of the energy function have been established by using Nakao's inequality. For more results about the Kirchhoff type equations we refer the readers to $[6,7,8,9,10]$.

On the other hand, it is known that modeling of some physical phenomena such as flows of electro-rheological fluids, nonlinear viscoelasticity and image processing give rise to equation with nonstandard growth conditions, that is, equations with variable exponents of nonlinearities. In [11], Shahrouzi and Kargarfard proved the blow-up result for the following Kirchhoff type problem:

$$
\begin{gathered}
u_{t t}-M\left(\|\nabla u\|^{2}\right) \Delta u-\Delta_{m(x)} u+h(x, t, u, \nabla u)+\beta u_{t}=\phi_{p(x)}(u), \text { in } \Omega \times(0,+\infty) \\
\left\{\begin{array}{cc}
u(x, t)=0, & (x, t) \in \Gamma_{0} \times(0,+\infty) \\
M\left(\|\nabla u\|^{2}\right) \frac{\partial u}{\partial n}(x, t)=\alpha u-|\nabla u|^{m(x)} \frac{\partial u}{\partial n}, & (x, t) \in \Gamma_{1} \times(0,+\infty)
\end{array}\right.
\end{gathered}
$$




$$
u(x, 0)=u_{0}(x), u_{t}(x, 0)=u_{1}(x), \text { in } \Omega,
$$

and proved the blow up of solutions with positive initial energy and suitable conditions on datas. Recently, Antontsev et. al [12], investigated the following nonlinear Timoshenko equation with variable exponents

$$
u_{t t}+\Delta^{2} u+M\left(\|\nabla u\|_{L^{2}(\Omega)}^{2}\right) \Delta u+\left|u_{t}\right|^{m(x)-2} u_{t}=|u|^{q(x)-2} u,
$$

and by using the Faedo-Galerkin method, they proved the local existence of the solution under suitable conditions. Also, the nonexistence of solutions with negative initial energy has been investigated. (see also [13, 14, 15])

Dai and Hao [16] studied the following problem

$$
\begin{gathered}
-M\left(\int_{\Omega} \frac{1}{p(x)}|\nabla u|^{p(x)} d x\right) \operatorname{div}\left(|\nabla u|^{p(x)-2} \nabla u\right)=f(x, u), \text { in } \Omega \\
u=0, \text { on } \partial \Omega .
\end{gathered}
$$

By means of a direct variational approach and the theory of the variable exponent Sobolev spaces, they established conditions ensuring the existence and multiplicity of solutions for the problem. Recently, Hamdani et. al. [17] investigated the following nonlocal $p(x)$-Kirchhoff problem:

$$
\begin{gathered}
-\left(a-b \int_{\Omega} \frac{1}{p(x)}|\nabla u|^{p(x)} d x\right) \operatorname{div}\left(|\nabla u|^{p(x)-2} \nabla u\right)=\lambda|u|^{p(x)-2} u+g(x, u), \text { in } \Omega \\
u=0, \text { on } \partial \Omega .
\end{gathered}
$$

They obtained a nontrivial weak solution by using the Mountain Pass theorem. For more results in Kirchhoff type equations with variable-exponents nonlinearities we refer the reader to [18, 19, 20, 21, 22, 23] and references therein.

Motivated by the aforementioned works, in the present paper, we study a $r(x)$ - Kirchhoff type equation with variableexponent nonlinearities. Under appropriate conditions on the initial data and variable exponents, we prove asymptotic stability and blow up of solutions with positive initial energy.

The rest of paper is organized as follows. In Section 2, we recall some definitions and Lemmas about the variable-exponent Lebesgue space, $L^{p(.)}(\Omega)$, the Sobolev space, $W^{1, p(.)}(\Omega)$ and additional conditions that be use for main results. In Section 3 , we prove the asymptotic stability of solutions for appropriate initial data and variable exponents. Finally, the blow-up result has been proved with positive initial energy and suitable conditions on data and variable exponents, in fourth Section.

\section{Preliminaries}

Throughout this work, all the functions considered are real-valued. We denote by $\|\cdot\|_{q}$ the $L^{q}$-norm over $\Omega$. In particular, the $L^{2}$-norm is denoted $\|$.$\| in \Omega$. In order to study problem (1.1)-(1.3), we need some theories about Lebesgue and Sobolev spaces with variable-exponents (for detailed, see [24, 25, 26, 27, 28]). Let $p(x) \geq 1$ and measurable, we assume that

$$
\begin{gathered}
C_{+}(\bar{\Omega})=\{h \mid h \in C(\bar{\Omega}), h(x)>1 \forall x \in \bar{\Omega}\}, \\
h^{+}=\max _{\bar{\Omega}} h(x), h^{-}=\min _{\bar{\Omega}} h(x) \text { for any } h \in C(\bar{\Omega}), \\
L^{p(x)}(\Omega)=\left\{u \mid \text { u is a measurable real - valued function, } \int_{\Omega}|u(x)|^{p(x)} d x<\infty\right\} .
\end{gathered}
$$

We equip the Lebesgue space with a variable exponent, $L^{p(x)}(\Omega)$, with the following Luxembourg-type norm

$$
\|u\|_{p(x)}:=\inf \left\{\lambda>\left.0\left|\int_{\Omega}\right| \frac{u(x)}{\lambda}\right|^{p(x)} d x \leq 1\right\}
$$

Lemma 2.1. [24, 28] Let $\Omega$ be a bounded domain in $R^{n}$

(i) the space $\left(L^{p(x)}(\Omega),\|\cdot\|_{p(x)}\right)$ is a Banach space, and its conjugate space is $L^{q(x)}(\Omega)$, where $\frac{1}{q(x)}+\frac{1}{p(x)}=1$. For any $u \in L^{p(x)}(\Omega)$ and $v \in L^{q(x)}(\Omega)$, we have

$$
\left|\int_{\Omega} u v d x\right| \leq\left(\frac{1}{p^{-}}+\frac{1}{q^{-}}\right)\|u\|_{p(x)}\|v\|_{q(x)} .
$$

(ii) If $p, q \in C_{+}(\bar{\Omega}), q(x) \leq p(x)$ for any $x \in \bar{\Omega}$, then $L^{p(x)}(\Omega) \hookrightarrow L^{q(x)}(\Omega)$, and the imbedding is continuous. 
The variable-exponent Lebesgue Sobolev space $W^{1, p(x)}(\Omega)$ is defined by

$$
W^{1, p(x)}(\Omega)=\left\{u \in L^{p(x)}(\Omega) \mid \nabla u \text { exists and }|\nabla u| \in L^{p(x)}(\Omega)\right\} .
$$

This space is a Banach space with respect to the norm $\|u\|_{W^{1, p(x)}(\Omega)}=\|u\|_{p(x)}+\|\nabla u\|_{p(x)}$. Furthermore, let $W_{0}^{1, p(x)}(\Omega)$ be the closure of $C_{0}^{\infty}(\Omega)$ in $W^{1, p(x)}(\Omega)$. The dual of $W_{0}^{1, p(x)}(\Omega)$ is defined as $W^{-1, p^{\prime}(x)}(\Omega)$, by the same way as the usual Sobolev spaces, where $\frac{1}{p(x)}+\frac{1}{p^{\prime}(x)}=1$.

If we define

$$
p^{*}(x)=\left\{\begin{array}{cc}
\frac{N p(x)}{N-p(x)}, & p^{+}<N \\
\infty, & p^{+} \geq N
\end{array}\right.
$$

then we have

Lemma 2.2. $[24,28]$ Let $\Omega$ be a bounded domain in $R^{n}$ then for any measurable bounded exponent $p(x)$ we have (i) $W^{1, p(x)}(\Omega)$ and $W_{0}^{1, p(x)}(\Omega)$ are separable Banach spaces;

(ii) if $q \in C_{+}(\bar{\Omega})$ and $q(x)<p^{*}(x)$ for any $x \in \bar{\Omega}$, then the imbedding $W^{1, p(x)}(\Omega) \hookrightarrow L^{q(x)}(\Omega)$ is compact and continuous;

(iii) if $p(x)$ is uniformly continuous in $\Omega$ then there exists a constant $C>0$, such that

$$
\|u\|_{p(x)} \leq C\|\nabla u\|_{p(x)} \quad \forall u \in W_{0}^{1, p(x)}(\Omega) .
$$

By (iii) of Lemma 2.2, we know that the space $W_{0}^{1, p(x)}(\Omega)$ has an equivalent norm given by $\|u\|_{W^{1, p(x)}(\Omega)}=\|\nabla u\|_{p(x)}$. We recall the trace Sobolev embedding in Lebesgue space with a constant exponent

$$
H_{\Gamma_{0}}^{1}(\Omega) \hookrightarrow L^{q}\left(\Gamma_{1}\right) \quad \text { for } \quad 2 \leq q<\frac{2(n-1)}{n-2}
$$

where

$$
H_{\Gamma_{0}}^{1}(\Omega)=\left\{u \in H^{1}(\Omega):\left.u\right|_{\Gamma_{0}}=0\right\}
$$

and the embedding inequality

$$
\|u\|_{q, \Gamma_{1}} \leq B_{q}\|\nabla u\|_{2}
$$

where $B_{q}$ is the optimal constant.

We sometimes use the Young's inequality

$$
a b \leq \beta a^{q}+C(\theta, q) b^{q^{\prime}}, a, b \geq 0, \quad \theta>0, \frac{1}{q}+\frac{1}{q^{\prime}}=1,
$$

where $C(\theta, q)=\frac{1}{q^{\prime}}(\theta q)^{-\frac{q^{\prime}}{q}}$ are constants.

\section{Asymptotic stability}

In this section we prove a stability result for the solution energy. For this goal we make the following assumptions:

(A2) There exist $\varepsilon>0$ sufficiently small and $\beta$ sufficiently large such that

$$
p_{2} \leq \frac{1}{\varepsilon(\beta-\varepsilon)} \leq r_{1} \leq r(x) \leq r_{2} \leq 2 \varepsilon(\beta-\varepsilon) r_{1}^{2} .
$$

The energy associated with problem (1.1)-(1.3) is given by

$$
E(t)=\frac{1}{2}\left(\left\|u_{t}\right\|^{2}+\|\nabla u\|^{2}\right)+\left(a+\frac{b}{2} \int_{\Omega} \frac{1}{r(x)}|\nabla u|^{r(x)} d x\right) \int_{\Omega} \frac{1}{r(x)}|\nabla u|^{r(x)} d x-\int_{\Omega} \frac{1}{p(x)}|u|^{p(x)} d x .
$$

Our main result in this section reads in the following theorem:

Theorem 3.1. Let the conditions (A1) and (A2) are satisfied. Then the energy E ( $t)$ of problem (1.1)-(1.3) tends to zero as time goes to infinity. 
To prove the above theorem, we need following Lemmas. First, we define

$$
F(t)=E(t)+\varepsilon \int_{\Omega} u u_{t} d x
$$

for some $\varepsilon>0$.

Lemma 3.2. Let $u$ be the solution of(1.1)-(1.3). Then the energy functional satisfies

$$
E^{\prime}(t)=-\beta \int_{\Omega}\left|u_{t}\right|^{2} d x \leq 0
$$

Proof. By multiplying equation (1.1) by $u_{t}$ and integrating over $\Omega$, using integration by parts, we obtain (3.2) for any regular solution. This equality remains valid for weak solutions by a simple density argument.

The following Lemma estimates an appropriate upper bound for $F^{\prime}(t)$ :

Lemma 3.3. Under the assumptions of Theorem 3.1, $F(t)$ satisfies, along the solution, the estimate

$$
F^{\prime}(t) \leq-(\beta-\varepsilon)\left\|u_{t}\right\|^{2}-\varepsilon\|\nabla u\|^{2}-\varepsilon \beta \int_{\Omega} u u_{t} d x-\varepsilon \int_{\Omega}|u|^{p(x)} d x-\varepsilon\left(a+b \int_{\Omega} \frac{1}{r(x)}|\nabla u|^{r(x)} d x\right) \int_{\Omega}|\nabla u|^{r(x)} d x .
$$

Proof. To prove this Lemma, at first we differentiate $F(t)$ to obtain

$$
F^{\prime}(t)=E^{\prime}(t)+\varepsilon\left\|u_{t}\right\|^{2}+\varepsilon \int_{\Omega} u u_{t t} d x
$$

thanks to Lemma 3.2, we get

$$
F^{\prime}(t) \leq-(\beta-\varepsilon)\left\|u_{t}\right\|^{2}+\varepsilon \int_{\Omega} u u_{t t} d x .
$$

By multiplying (1.1) in $u$, it is easy to see that

$$
\int_{\Omega} u u_{t t} d x=-\|\nabla u\|^{2}-\left(a+b \int_{\Omega} \frac{1}{r(x)}|\nabla u|^{r(x)} d x\right) \int_{\Omega}|\nabla u|^{r(x)} d x-\beta \int_{\Omega} u u_{t} d x+\int_{\Omega}|u|^{p(x)} d x .
$$

Combining (3.5) with (3.4), proof is completed.

Now, from definition of $F(t)$ and Lemma 3.3, we have

$$
\begin{aligned}
& F^{\prime}(t)+\frac{1}{(\beta-\varepsilon)} F(t) \leq-\left(\beta-\frac{1}{2(\beta-\varepsilon)}-\varepsilon\right)\left\|u_{t}\right\|^{2}-\left(\varepsilon-\frac{1}{2(\beta-\varepsilon)}\right)\|\nabla u\|^{2}-a\left(\varepsilon-\frac{1}{r_{1}(\beta-\varepsilon)}\right) \int_{\Omega}|\nabla u|^{r(x)} d x \\
& -b\left(\frac{\varepsilon}{r_{2}}-\frac{1}{2 r_{1}^{2}(\beta-\varepsilon)}\right)\left(\int_{\Omega}|\nabla u|^{r(x)} d x\right)^{2}-\left(\frac{1}{p_{2}(\beta-\varepsilon)}-\varepsilon\right) \int_{\Omega}|u|^{p(x)} d x-\int_{\Omega} u u_{t} d x .
\end{aligned}
$$

Using the Young and Poincaré inequalities, we get

$$
\left|\int_{\Omega} u u_{t} d x\right| \leq \frac{\varepsilon}{2}\|\nabla u\|^{2}+\frac{B_{2}^{2}}{2 \varepsilon}\left\|u_{t}\right\|^{2}
$$

where $B_{2}$ is the best constant in Poincaré inequality.

Utilizing (3.7) into (3.6) to get

$$
\begin{aligned}
& F^{\prime}(t)+\frac{1}{(\beta-\varepsilon)} F(t) \leq-\left(\beta-\frac{1}{2(\beta-\varepsilon)}-\varepsilon-\frac{B_{2}^{2}}{2 \varepsilon}\right)\left\|u_{t}\right\|^{2}-\left(\frac{\varepsilon}{2}-\frac{1}{2(\beta-\varepsilon)}\right)\|\nabla u\|^{2}-a\left(\varepsilon-\frac{1}{r_{1}(\beta-\varepsilon)}\right) \int_{\Omega}|\nabla u|^{r(x)} d x \\
& \quad-b\left(\frac{\varepsilon}{r_{2}}-\frac{1}{2 r_{1}^{2}(\beta-\varepsilon)}\right)\left(\int_{\Omega}|\nabla u|^{r(x)} d x\right)^{2}-\left(\frac{1}{p_{2}(\beta-\varepsilon)}-\varepsilon\right) \int_{\Omega}|u|^{p(x)} d x .
\end{aligned}
$$

Thanks to the (A2), we deduce

$$
F^{\prime}(t)+\frac{1}{(\beta-\varepsilon)} F(t) \leq 0 .
$$

Integrating over $(0, \mathrm{t})$, we get from (3.9)

$$
F(t) \leq F(0) e^{\frac{-t}{\beta-\varepsilon}},
$$

according to $(A 2)$, this inequality show that $F(t) \rightarrow 0$ as $t \rightarrow \infty$. Since $E(t) \leq C_{0} F(t)$, thus the proof of Theorem 3.1 has been completed. 


\section{Blow-up}

In this section we are going to prove that for appropriate initial data some of the solutions blow up in a finite time. To prove the blow-up result for certain solutions with positive initial energy, we assumed that:

(A3)

$$
p_{1} \geq r_{2}+2, \quad r_{2}^{2} \leq 2 r_{1}^{2} \leq 2 r_{2}^{2}
$$

At this point, we shall add a new variable $v(x, t)$ to the system (1.1)-(1.3). Let us define for any $\lambda>0$

$$
v(x, t)=e^{-\lambda t} u(x, t) .
$$

A direct computation by substituting (4.1) into the problem (1.1)-(1.3), yields

$$
\begin{gathered}
v_{t t}+(2 \lambda+\beta) v_{t}+\lambda(\lambda+\beta) v-\Delta v-\left(a+b \int_{\Omega} \frac{1}{r(x)}\left|e^{\lambda t} \nabla v\right|^{r(x)} d x\right) d i v\left(\left|e^{\lambda t} \nabla v\right|^{r(x)-2} \nabla v\right)=\left|e^{\lambda t} v\right|^{p(x)-2} v, \quad(x, t) \in \Omega \times(0, \infty) \\
v(x, t)=0, \quad(x, t) \in \partial \Omega \times(0, \infty), \\
v(x, 0)=u_{0}(x), \quad v_{t}(x, 0)=u_{1}(x)-\lambda u_{0}(x), \quad x \in \Omega .
\end{gathered}
$$

The energy function related with problem (4.2)-(4.4) is given by

$$
E_{\lambda}(t)=e^{-2 \lambda t} \int_{\Omega} \frac{\left|e^{\lambda t} v\right|^{p(x)}}{p(x)} d x-\frac{1}{2} I(t)
$$

where

$$
I(t)=\left\|v_{t}\right\|^{2}+\lambda(\lambda+\beta)\|v\|^{2}+\|\nabla v\|^{2}+2 a e^{-2 \lambda t} \int_{\Omega} \frac{\left|e^{\lambda t} \nabla v\right|^{r(x)}}{r(x)} d x+b e^{-2 \lambda t}\left(\int_{\Omega} \frac{\left|e^{\lambda t} \nabla v\right|^{r(x)}}{r(x)} d x\right)^{2}
$$

Now we are in a position to state our blow-up result as follows:

Theorem 4.1. Let the conditions (A1) and (A3) are satisfied. Assume that $E_{\lambda}(0)>0$. Then there exists a finite time $t^{*}$ such that the solution of the problem (1.1)-(1.3) blows up in a finite time, that is

$$
\|u(t)\| \rightarrow+\infty \text { as } t \rightarrow t^{*} .
$$

To prove the blow-up result, we need the following Lemma.

Lemma 4.2. Under the conditions of Theorem 4.1, the energy functional $E_{\lambda}(t)$, defined by (4.5), satisfies

$$
E_{\lambda}(t) \geq e^{r_{2} \lambda t} E_{\lambda}(0) \quad \forall t \in R^{+} .
$$

Proof. A multiplication of equation (4.2) by $v_{t}$ and integrating over $\Omega$ gives

$$
\begin{gathered}
E_{\lambda}^{\prime}(t)=(2 \lambda+\beta)\left\|v_{t}\right\|^{2}-b \lambda e^{-2 \lambda t}\left(\int_{\Omega} \frac{\left|e^{\lambda t} \nabla v\right|^{r(x)}}{r(x)} d x\right)^{2}-a e^{-2 \lambda t} \int_{\Omega} \frac{\lambda(r(x)-2)}{r(x)}\left|e^{\lambda t} \nabla v\right|^{r(x)} d x \\
+e^{-2 \lambda t} \int_{\Omega} \frac{\lambda(p(x)-2)}{p(x)}\left|e^{\lambda t} v\right|^{p(x)} d x-b e^{-2 \lambda t}\left(\int_{\Omega} \frac{\left|e^{\lambda t} \nabla v\right|^{r(x)}}{r(x)} d x\right)\left(\int_{\Omega} \frac{\lambda(r(x)-2)}{r(x)}\left|e^{\lambda t} \nabla v\right|^{r(x)} d x\right),
\end{gathered}
$$

next, for any $\varepsilon>0$, we have

$$
\begin{aligned}
& E_{\lambda}^{\prime}(t)-\varepsilon E_{\lambda}(t)=\left(2 \lambda+\beta+\frac{\varepsilon}{2}\right)\left\|v_{t}\right\|^{2}+b\left(\frac{\varepsilon}{2}-\lambda\right) e^{-2 \lambda t}\left(\int_{\Omega} \frac{\left|e^{\lambda t} \nabla v\right|^{r(x)}}{r(x)} d x\right)^{2}+\frac{\varepsilon}{2} \lambda(\lambda+\beta)\|v\|^{2}+\frac{\varepsilon}{2}\|\nabla v\|^{2} \\
& \quad+a \varepsilon e^{-2 \lambda t} \int_{\Omega} \frac{\left|e^{\lambda t} \nabla v\right|^{r(x)}}{r(x)} d x-a e^{-2 \lambda t} \int_{\Omega} \frac{\lambda(r(x)-2)}{r(x)}\left|e^{\lambda t} \nabla v\right|^{r(x)} d x+e^{-2 \lambda t} \int_{\Omega} \frac{\lambda(p(x)-2)}{p(x)}\left|e^{\lambda t} v\right|^{p(x)} d x
\end{aligned}
$$




$$
-\varepsilon e^{-2 \lambda t} \int_{\Omega} \frac{\left|e^{\lambda t} v\right|^{p(x)}}{p(x)} d x-b e^{-2 \lambda t}\left(\int_{\Omega} \frac{\left|e^{\lambda t} \nabla v\right|^{r(x)}}{r(x)} d x\right)\left(\int_{\Omega} \frac{\lambda(r(x)-2)}{r(x)}\left|e^{\lambda t} \nabla v\right|^{r(x)} d x\right) .
$$

Utilizing additional condition $(A 1)$, we get

$$
\begin{gathered}
E_{\lambda}^{\prime}(t)-\varepsilon E_{\lambda}(t) \geq b\left(\frac{\varepsilon}{r_{2}^{2}}-\frac{\lambda\left(r_{2}-2\right)}{r_{2}^{2}}-\frac{\lambda}{r_{1}^{2}}\right) e^{-2 \lambda t}\left(\int_{\Omega}\left|e^{\lambda t} \nabla v\right|^{r(x)} d x\right)^{2}+a\left(\frac{\varepsilon}{r_{2}}-\frac{\lambda\left(r_{2}-2\right)}{r_{2}}\right) e^{-2 \lambda t} \int_{\Omega}\left|e^{\lambda t} \nabla v\right|^{r(x)} d x \\
+\frac{1}{p_{1}}\left(\lambda\left(p_{1}-2\right)-\varepsilon\right) e^{-2 \lambda t} \int_{\Omega}\left|e^{\lambda t} v\right|^{p(x)} d x .
\end{gathered}
$$

Finally, if we set $\varepsilon:=r_{2} \lambda$ then by using $(A 3)$ we arrive at

$$
E_{\lambda}^{\prime}(t)-\varepsilon E_{\lambda}(t) \geq 0
$$

and by integration over $(0, t)$ we obtain the desired result.

Proof of Theorem 4.1. For obtaining the blow-up result, the choice of the following functional is standard

$$
\psi(t)=\|v(t)\|^{2},
$$

then

$$
\begin{aligned}
& \psi^{\prime}(t)=2 \int_{\Omega} v v_{t} d x, \\
& \psi^{\prime \prime}(t)=2 \int_{\Omega} v v_{t t} d x+2\left\|v_{t}\right\|^{2} .
\end{aligned}
$$

A multiplication of equation (4.2) by $v$ and integrating over $\Omega$ gives

$$
\begin{aligned}
& \int_{\Omega} v_{t t} v d x=-(2 \lambda+\beta) \int_{\Omega} v v_{t} d x-\lambda(\lambda+\beta)\|v\|^{2}-\|\nabla v\|^{2}-e^{-2 \lambda t}\left(a+b \int_{\Omega} \frac{\left|e^{\lambda t} \nabla v\right|^{r(x)}}{r(x)} d x\right) \int_{\Omega}\left|e^{\lambda t} \nabla v\right|^{r(x)} d x . \\
& +e^{-2 \lambda t} \int_{\Omega}\left|e^{\lambda t} v\right|^{p(x)} d x
\end{aligned}
$$

By using definition of $E_{\lambda}(t)$ in (4.11), we have

$$
\begin{aligned}
\int_{\Omega} v_{t t} v d x \geq r_{2} E_{\lambda}(t)+\frac{r_{2}}{2}\left\|v_{t}\right\|^{2} & +\lambda\left(\frac{r_{2}}{2}-1\right)(\lambda+\beta)\|v\|^{2}+\left(\frac{r_{2}}{2}-1\right)\|\nabla v\|^{2}+\frac{b\left(r_{1}-2\right)}{2 r_{1}} e^{-2 \lambda t}\left(\int_{\Omega}\left|e^{\lambda t} \nabla v\right|^{r(x)} d x\right)^{2} \\
& +\left(1-\frac{r_{2}}{p_{1}}\right) e^{-2 \lambda t} \int_{\Omega}\left|e^{\lambda t} v\right|^{p(x)} d x-(2 \lambda+\beta) \int_{\Omega} v v_{t} d x
\end{aligned}
$$

and taking into account $(A 3)$ to obtain

$$
\int_{\Omega} v_{t t} v d x \geq r_{2} E_{\lambda}(t)+\frac{r_{2}}{2}\left\|v_{t}\right\|^{2}+\lambda\left(\frac{r_{2}}{2}-1\right)(\lambda+\beta)\|v\|^{2}-(2 \lambda+\beta) \int_{\Omega} v v_{t} d x .
$$

By substituting (4.8)-(4.10) in (4.12) we get

$$
\begin{aligned}
\psi^{\prime \prime}(t) & \geq 2 r_{2} E_{\lambda}(t)+\left(r_{2}+2\right)\left\|v_{t}\right\|^{2}+\lambda(\lambda+\beta)\left(r_{2}-2\right) \psi(t)-(2 \lambda+\beta) \psi^{\prime}(t) \\
& \geq\left(r_{2}+2\right)\left\|v_{t}\right\|^{2}+\lambda(\lambda+\beta)\left(r_{2}-2\right) \psi(t)-(2 \lambda+\beta) \psi^{\prime}(t),
\end{aligned}
$$

where Lemma 4.2 and hypotheses of Theorem 4.1 about initial energy have been used. Multiplying (4.13) in $\psi(t)$, we get

$$
\psi(t) \psi^{\prime \prime}(t) \geq\left(r_{2}+2\right)\|v\|^{2}\left\|v_{t}\right\|^{2}+\lambda(\lambda+\beta)\left(r_{2}-2\right) \psi^{2}(t)-(2 \lambda+\beta) \psi(t) \psi^{\prime}(t),
$$


and finally we obtain

$$
\psi(t) \psi^{\prime \prime}(t) \geq \frac{\left(r_{2}+2\right)}{4}\left(\psi^{\prime}(t)\right)^{2}+\lambda(\lambda+\beta)\left(r_{2}-2\right) \psi^{2}(t)-(2 \lambda+\beta) \psi(t) \psi^{\prime}(t),
$$

where the inequality $\left(\psi^{\prime}(t)\right)^{2} \leq 4\|v\|^{2}\left\|v_{t}\right\|^{2}$ has been used.

Thus by the modified concavity method we deduce that there exists a finite time $t^{*}$ such that

$$
\lim _{t \rightarrow t^{*}} \psi(t)=\infty
$$

Consequently, the proof of Theorem 4.1 has been completed.

\section{References}

[1] G. Kirchhoff, Vorlesungen uber Mechanik, Teubner, Leipzig , (1883).

[2] T. Matsuyama, R. Ikehata, On global solutions and energy decay for the wave equations of Kirchhoff type with nonlinear damping terms, J. Math. Anal. Appl. 204, 729-753 (1996).

[3] K. Ono, On global solutions and blow-up solutions of nonlinear Kirchhoff strings with nonlinear dissipation, J. Math. Anal. Appl. 216, 321-342 (1997).

[4] E. Pişkin, Existence, decay and blow up of solutions for the extensible beam equation with nonlinear damping and source terms, Open Math. 13, 408-420 (2015).

[5] J. Zhou, Global existence and blow-up of solutions for a Kirchhoff type plate equation with damping, Appl. Math. Comput. 265, 807-818 (2015).

[6] R. Ikehata, A note on the global solvability of solutions to some nonlinear wave equations with dissipative terms, Differ. Integral Equ. 8, 607-616 (1995).

[7] S. A. Messaoudi, Blow-up of solutions for the Kirchhoff equation of $q$-Laplacian type with nonlinear dissipation, Colloq. Math. 94, 103-109 (2002).

${ }^{[8]} \mathrm{K}$. Ono, On global existence, asymptotic stability and blowing up of solutions for some degenerate nonlinear wave equations of Kirchhoff type with a strong dissipation, Math. Meth. Appl. Sci. 20, 151-177 (1997).

${ }^{[9]}$ C. O. Alves, F. J. S. A. Corrêa, T. F. Ma, Positive Solutions for a Quasilinear Elliptic Equation of Kirchhoff Type, Comput. Math. Appl. 49, 85-93 (2005).

[10] A. Yang, Z. Gong, Blow-up of solutions for some nonlinear wave equations of Kirchhoff type with arbitrary positive initial energy, Electron. J. Differ. Equ. 332, 1-8 (2016).

[11] M. Shahrouzi, F. Kargarfard, Blow-up of solutions for a Kirchhoff type equation with variable-exponent nonlinearities, J. App. Anal. 27(1), 97-105 (2021).

${ }^{[12]}$ S. N. Antontsev, J. Ferreira, E. Pişkin, S. M. S. Cordeiro, Existence and non-existence of solutions for Timoshenko-type equations with variable exponents, Nonlinear Anal. Real World Appl. 61, 103341, (2021).

[13] E. Pişkin, Finite time blow up of solutions of the Kirchhoff-type equation with variable exponents, Int. J. Nonlinear Anal. Appl. 11(1), 37-45 (2020).

[14] M. Shahrouzi, Blow up of solutions for a $r(x)$-Laplacian Lamé equation with variable-exponent nonlinearities and arbitrary initial energy level, Int. J. Nonlinear Anal. Appl. 13(1), 441-450 (2022).

[15] M. Shahrouzi, General decay and blow up of solutions for a class of inverse problem with elasticity term and variableexponent nonlinearities, Math. Meth. Appl. Sci. 2021. DOI: https://doi.org/10.1002/mma.7891.

[16] G. Dai, R. Hao, Existence of solutionsfor a p (x)-Kirchhoff-type equation, J. Math. Anal. Appl. 359, 275-284 (2009).

[17] M. K. Hamdani, A. Harrabi, F. Mtiri, D. D. Repově, Existence and multiplicity results for a new $p(x)-$ Kirchhoff problem, Nonlinear Analysis 190, 111598 (2020).

[18] G. Dai, R. Ma, Solutions for a $p(x)$-Kirchhoff type equation with Neumann boundary data, Nonlinear Analysis: Real World Applications 12 , 2666-2680 (2011).

[19] A. Ghanmi, Nontrivial solutions for Kirchhoff-type problems involving the $p(x)$-Laplace operator, Rocky Mount. J. Math. 48(4), 1145-1158 (2018). 
[20] E. J. Hurtado, O. H. Miyagaki, R. D. S. Rodrigues, Existence and Asymptotic Behaviour for a Kirchhoff Type Equation With Variable Critical Growth Exponent, Milan J. Math. 85, 71-102 (2017).

[21] S. Heidarkhani, A. L. A. De Araujo, G. A. Afrouzi, S. Moradi, Multiple solutions for Kirchhoff-type problems with variable exponent and nonhomogeneous Neumann conditions, Math. Nachr. 291(2), 326-342 (2018).

[22] M. Shahrouzi, On behaviour of solutions for a nonlinear viscoelastic equation with variable-exponent nonlinearities, Comp. Math. Appl. 75, 3946-3956 (2018).

[23] M. Shahrouzi, Global nonexistence of solutions for a class of viscoelastic Lamé system, Indian J. Pure Appl. Math. 51(4), 1383-1397 (2020).

[24] L. Diening, P. Hasto, et al. Lebesgue and Sobolev spaces with variable exponents, Springer-Verlag, (2011).

[25] D. Edmunds, J. Rakosnik, Sobolev embeddings with variable exponent, Stud. Math. 143, 267-293 (2000).

[26] D. Edmunds, J. Rakosnik, Sobolev embeddings with variable exponent II, Math. Nachr. 246, 53-67 (2002).

[27] X. Fan, D. Zhao, On the spaces $L^{p(x)}$ and $W^{m, p(x)}(\Omega)$, J. Math. Anal. Appl. 263, 424-446 (2001).

[28] X. Fan, Q. H. Zhang, Existence of solutions for $p(x)$-Laplacian Dirichlet problem, Nonlinear Anal. 52, 1843-1852 (2003). 